\title{
PAPER
}

\section{Cerebral vasomotor reactivity testing in head injury: the link between pressure and flow}

\author{
E W Lang, J Lagopoulos, J Griffith, K Yip, A Yam, Y Mudaliar, H M Mehdorn, \\ N W C Dorsch
}

See end of article for authors' affiliations .....................

Correspondence to: Dr Erhard W Lang, Department of

Neurosurgery, Weimarer Strasse 8, D-24106 Kiel, Germany

Received

18 December 2002

In revised form 6 April

2003

Accepted 11 April 2003
Background: It has been suggested that a moving correlation index between mean arterial blood pressure and intracranial pressure, called PRx, can be used to monitor and quantify cerebral vasomotor reactivity in patients with head injury.

Objectives: To validate this index and study its relation with cerebral blood flow velocity and cerebral autoregulation; and to identify variables associated with impairment or preservation of cerebral vasomotor reactivity.

Methods: The PRx was validated in a prospective study of 40 head injured patients. A PRx value of less than 0.3 indicates intact cerebral vasomotor reactivity, and a value of more than 0.3 , impaired reactivity. Arterial blood pressure, intracranial pressure, mean cerebral perfusion pressure, and cerebral blood flow velocity, measured bilaterally with transcranial Doppler ultrasound, were recorded. Dynamic cerebrovascular autoregulation was measured using a moving correlation coefficient between arterial blood pressure and cerebral blood flow velocity, the $M x$, for each cerebral hemisphere. All variables were compared in patients with intact and impaired cerebral vasomotor reactivity.

Results: No correlation between arterial blood pressure or cerebral perfusion pressure and cerebral blood flow velocity was seen in 19 patients with intact cerebral vasomotor reactivity. In contrast, the correlation between these variables was significant in 21 patients with impaired cerebral vasomotor reactivity, whose cerebral autoregulation was reduced. There was no correlation with intracranial pressure, arterial blood pressure, cerebral perfusion pressure, or interhemispheric cerebral autoregulation differences, but the values for these indices were largely within normal limits.

Conclusions: The PRx is valid for monitoring and quantifying cerebral vasomotor reactivity in patients with head injury. This intracranial pressure based index reflects changes in cerebral blood flow and cerebral autoregulatory capacity, suggesting a close link between blood flow and intracranial pressure in head injured patients. This explains why increases in arterial blood pressure and cerebral perfusion pressure may be useful for reducing intracranial pressure in selected head injured patients (those with intact cerebral vasomotor reactivity).
$\mathrm{S}$ pontaneous or pharmacologically induced variations in arterial blood pressure or cerebral perfusion pressure can have different effects on intracranial pressure depending on so called "cerebral vasomotor reactivity" or "cerebrovascular reactivity." We have anecdotally reported that increasing the cerebral perfusion pressure can lead to either an increase or a decrease in intracranial pressure, depending on the individual vascular regulatory capacity (cerebral pressure autoregulation). ${ }^{12}$ Similar evidence was provided by Rosner, who described the linkage between cerebral perfusion pressure decreases and intracranial pressure increases as the "vasodilatory cascade." ${ }^{\prime 3}$ In his model increases in cerebral perfusion pressure within the operative range of cerebral autoregulation cause compensatory active vasoconstriction to maintain a stable cerebral blood flow. This vasoconstriction leads to a decrease in cerebral blood volume and thereby to a decrease in intracranial pressure (fig 1). Below the lower cerebral autoregulation limit or with autoregulatory failure, an increase in cerebral perfusion pressure will result in passive vasodilatation which will increase the cerebral blood volume and therefore the intracranial pressure. In this situation of cerebral autoregulatory failure, cerebral blood flow will vary with cerebral perfusion pressure, and a stable blood flow can no longer be maintained (fig 2).

To quantify this variable relation between arterial blood pressure or cerebral perfusion pressure and intracranial pressure-cerebrovascular reactivity-the Cambridge group has defined an index comparing arterial blood pressure and intracranial pressure, the "Prx". ${ }^{4}$ This index illustrates the correlation between arterial blood pressure and intracranial pressure. If intracranial pressure follows arterial blood pressure in a parallel fashion, there is a good correlation, and the PRx index is positive. On the other hand, if an arterial blood pressure increase causes vasoconstriction (that is, pressure autoregulation is preserved), a reduction in cerebral blood volume, and a decrease in intracranial pressure, the positive correlation will be lost; in this case the PRx will approach zero or even become negative, indicating well preserved cerebrovascular reactivity. Although this explanation appears quite plausible, based as it is on current physiological models and frequent clinical observations, the presumed vascular mechanism linking cerebral blood flow or cerebral blood volume with intracranial pressure has never been systematically validated in a prospective clinical study in head injured patients.

To validate the cerebrovascular reactivity index and to study its relation with cerebral blood flow velocity and cerebral autoregulation, we examined the relation between cerebrovascular reactivity and the cerebral blood flow velocity, assessed by transcranial Doppler ultrasound. Our hypothesis was that there would not be a correlation between arterial blood pressure/cerebral perfusion pressure and cerebral blood flow velocity in patients with intact cerebrovascular reactivity, 

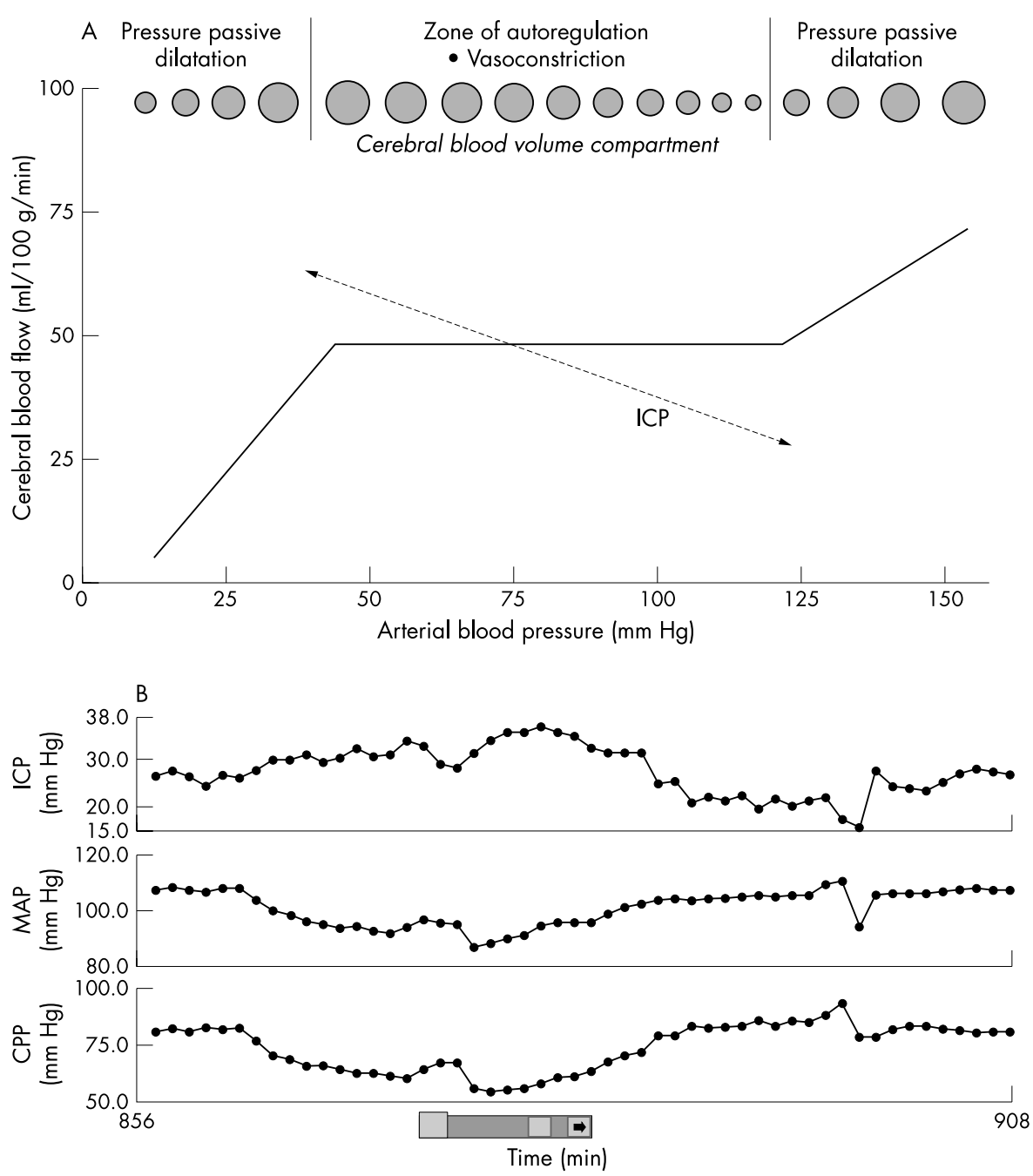

Figure 1 (A) A physiological model of intact cerebral autoregulation where, within the autoregulatory range between 50 and $150 \mathrm{~mm} \mathrm{Hg}$ arterial blood pressure (x axis), cerebral blood flow (y axis) remains stable. Blood pressure and cerebral blood flow are not correlated in this range. (B) Example of intact cerebral autoregulation recorded in a patient after traumatic brain injury. Intracranial pressure increases with decreasing blood pressure. When blood pressure is restored, intracranial pressure starts to decrease. CPP, cerebral perfusion pressure; ICP, intracranial pressure; MAP, mean arterial blood pressure. (ICP, MAP, and CPP in $\mathrm{mm} \mathrm{Hg}$ on the $y$ axis; time in minutes on the $x$ axis.)

whereas a linear correlation would be expected in those with defective cerebrovascular reactivity.

To examine the link between cerebrovascular reactivity and cerebral autoregulation we have used an index of cerebral autoregulation, the "Mx", which is a moving correlation coefficient between arterial blood pressure or cerebral perfusion pressure and cerebral blood flow velocity. ${ }^{5}$ This index can be calculated for each cerebral hemisphere. According to our hypothesis, cerebral autoregulation should be less effective in patients with impaired cerebrovascular reactivity than in those with intact reactivity.

We were also interested to identify any variables-arterial blood pressure, cerebral perfusion pressure, intracranial pressure, or cerebral blood flow velocity-associated with impairment or preservation of the cerebrovascular reactivity.

\section{METHODS}

\section{Patients}

The PRx was validated in a prospective study in 40 severely head injured patients, defined as having a Glasgow coma scale (GCS) score of 8 or less after initial resuscitation, or a deterioration to this level during the first 12 hours of treatment. The mean admission GCS was 8, and the mean (SD) age was 40 (16) years. There were eight female and 32 male patients. More details are given in table 1.
Blood pressure recordings were obtained with a radial artery fluid coupled system (pvb, Kirchseeon, Germany). Intracranial pressure was measured with an intraparenchymal sensor (Camino V420®, San Diego, California, USA, or Spiegelberg BrainPressure Monitor ${ }^{\circledR}$, Spiegelberg KG, Hamburg, Germany), or through an external ventricular drain. Intraparenchymal pressure sensors were placed intracranially on the side of injury or in the right frontal area if there was diffuse injury or multiple contusions, and external ventricular drains through a right frontal burr hole. Cerebral blood flow velocities were recorded bilaterally using transcranial Doppler ultrasound (Multi-Dop T® or Multi-Dop X2 ${ }^{\circledR}$, DWL, Sipplingen, Germany). All transcranial Doppler ultrasound studies were consistently performed by one of two examiners (EWL or $\mathrm{JG}$ ). All analogue signals were recorded and stored digitally in the transcranial Doppler ultrasound unit.

Management of these patients consisted of aggressive surgical and medical treatment including immediate evacuation of intracranial mass lesions, mechanical ventilation, and control of intracranial pressure, using a protocol consistent with the Guidelines for the Management of Severe Head Injury. ${ }^{6}$

The need for informed consent was waived because this was a non-invasive study, using routinely monitored variables and without any external stimulation. 

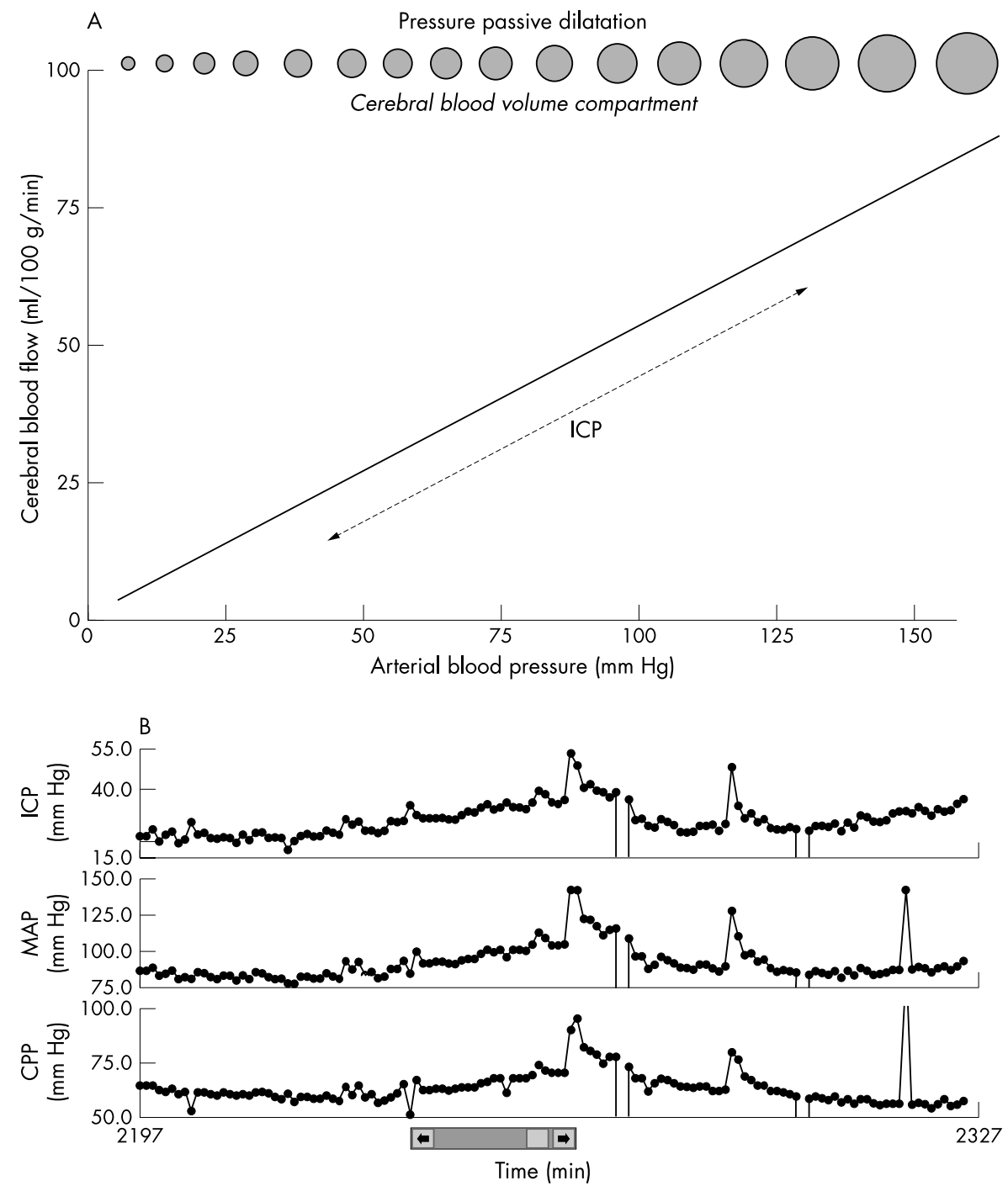

Figure 2 (A) Impaired cerebral autoregulation with flow (y axis) entirely dependent on blood pressure (x axis). There is a good correlation between flow and blood pressure. (B) An example of impaired cerebral autoregulation in a patient with traumatic brain injury. The intracranial pressure increases with increasing blood pressure, and falls when blood pressure decreases. CPP, cerebral perfusion pressure; ICP, intracranial pressure; MAP, mean arterial blood pressure. (ICP, MAP, and CPP in $\mathrm{mm} \mathrm{Hg}$ on the $y$ axis; time in minutes on the $x$ axis.)

\section{METHODS}

Cerebrovascular reactivity was determined using the PRx, which was calculated as a moving correlation coefficient between arterial blood pressure and intracranial pressure. ${ }^{4}$ Based upon this and other studies, a PRx value of more than 0.3 indicates impaired cerebrovascular reactivity and a value less than 0.3 , intact reactivity. ${ }^{7}$

Cerebral autoregulation was determined using the $\mathrm{Mx}$ for each cerebral hemisphere, comprising a moving correlation coefficient between arterial blood pressure and cerebral blood flow velocity. The Mx can be calculated from arterial blood pressure and cerebral perfusion pressure. In their original publication about the $\mathrm{Mx}$, the Cambridge group used cerebral perfusion pressure. ${ }^{5}$ In a more recent study they used arterial blood pressure for Mx calculations in healthy volunteers. ${ }^{8}$ In the present study we used arterial blood pressure for Mx calculation because first, intracranial pressure was largely within normal limits, and second, there were no large intracranial pressure fluctuations during the time of recording. The mean of left and right Mx values was used for comparison of cerebrovascular reactivity and cerebral autoregulation. Both the PRx and the Mx were calculated from 10000 simultaneously recorded data points, sampled at $57.4 \mathrm{~Hz}$ in each of the 40 patients on one occasion during the first five days of treat- ment, using spontaneous fluctuations in arterial blood pressure without the need for any induced change.

To examine whether cerebral autoregulation differs in patients with intact cerebrovascular reactivity compared with patients with impaired reactivity, we calculated the Mx for each group. To assess the overall relation between cerebrovascular reactivity and cerebral autoregulation we studied the correlation between the two indices. The absolute (numeric) left-right $\mathrm{Mx}$ difference $(\Delta \mathrm{l} / \mathrm{r} \mathrm{Mx}$ ) was used to examine whether there was any difference in hemispheric cerebral autoregulation asymmetry between the two groups.

To study whether any of the other monitored variablesarterial blood pressure, cerebral perfusion pressure, intracranial pressure, or cerebral blood flow velocity-was associated with impairment of cerebrovascular reactivity, we compared these variables between the two groups.

\section{RESULTS}

Cerebrovascular reactivity, as assessed by the $\mathrm{PRx}$, was impaired in 21 patients and intact in 19. In the 21 patients with impaired cerebrovascular reactivity there was a significant correlation between arterial blood pressure and cerebral blood flow velocity (Pearson correlation, $\mathrm{p}<0.01$; Spearman 


\begin{tabular}{|c|c|c|c|c|c|c|}
\hline Patient & GCS & Age (years) & Sex & GOS & Recording day & Injury \\
\hline 1 & 4 & 46 & $M$ & 4 & 4 & EDH, contusion \\
\hline 2 & 12 & 61 & $M$ & 5 & 4 & aSDH, contusion \\
\hline 3 & 11 & 28 & $M$ & 5 & 5 & $\mathrm{aSDH}$ \\
\hline 4 & 6 & 71 & $M$ & 1 & 0 & Contusion \\
\hline 5 & 14 & 63 & $M$ & 5 & 1 & $\mathrm{aSDH}$ \\
\hline 6 & 10 & 22 & $M$ & 5 & 1 & $\mathrm{EDH}$ \\
\hline 7 & 14 & 46 & $M$ & 4 & 2 & Contusion \\
\hline 8 & 14 & 44 & $M$ & 5 & 5 & Contusion \\
\hline 9 & 11 & 53 & $M$ & 3 & 4 & Contusion, aSDH, iSDH \\
\hline 10 & 11 & 18 & $\mathrm{~F}$ & 5 & 2 & Contusion, aSDH \\
\hline 11 & 7 & 44 & M & 5 & 5 & aSDH \\
\hline 12 & 4 & 17 & M & 2 & 1 & Brain stem contusion \\
\hline 13 & 10 & 28 & $M$ & 4 & 3 & $\mathrm{aSDH}$ \\
\hline 14 & 7 & 78 & $\mathrm{~F}$ & 1 & 1 & $\mathrm{aSDH}$ \\
\hline 15 & 14 & 36 & $M$ & 5 & 3 & $\mathrm{EDH}$ \\
\hline 16 & 13 & 59 & $M$ & 4 & 2 & $\mathrm{aSDH}$ \\
\hline 17 & 6 & 39 & $\mathrm{~F}$ & 1 & 2 & $\mathrm{aSDH}$ \\
\hline 18 & 12 & 47 & $M$ & 3 & 1 & Contusion \\
\hline 19 & 6 & 63 & M & 1 & 1 & $\mathrm{aSDH}$ \\
\hline 20 & 9 & 42 & M & 3 & 3 & $\mathrm{aSDH}$ \\
\hline 21 & 9 & 35 & M & 3 & 1 & Contusion \\
\hline 22 & 3 & 49 & M & 3 & 3 & Contusion \\
\hline 23 & 3 & 43 & M & 2 & 2 & Contusion, aSDH, EDH \\
\hline 24 & 12 & 58 & M & 5 & 2 & Contusion, aSDH \\
\hline 25 & 3 & 47 & $M$ & 1 & 1 & Contusion, aSDH \\
\hline 26 & 13 & 21 & M & 5 & 1 & Contusion \\
\hline 27 & 13 & 40 & M & 4 & 3 & $\mathrm{aSDH}, \mathrm{EDH}$ \\
\hline 28 & 3 & 26 & $\mathrm{~F}$ & 5 & 3 & $\mathrm{aSDH}$ \\
\hline 29 & 5 & 23 & $\mathrm{~F}$ & 4 & 1 & $\mathrm{DI}$ \\
\hline 30 & 6 & 43 & $M$ & 5 & 2 & Contusion, aSDH \\
\hline 31 & 15 & 30 & $\mathrm{~F}$ & 5 & 0 & $\mathrm{DI}$ \\
\hline 32 & 5 & 49 & $\mathrm{~F}$ & 3 & 3 & Contusion, aSDH \\
\hline 33 & 5 & 22 & $M$ & 2 & 3 & aSDH \\
\hline 34 & 3 & 24 & $M$ & 3 & 1 & $\mathrm{aSDH}$ \\
\hline 35 & 3 & 44 & $M$ & 1 & 1 & aSDH \\
\hline 36 & 6 & 16 & $\mathrm{~F}$ & 4 & 2 & Contusion, aSDH \\
\hline 37 & 7 & 18 & $M$ & 5 & 0 & $\mathrm{DI}$ \\
\hline 38 & 5 & 23 & $M$ & 5 & 0 & Contusion, EDH \\
\hline 39 & 3 & 46 & $M$ & 3 & 2 & Contusion, DI, IVH \\
\hline 40 & 4 & 52 & $M$ & 3 & 1 & Contusion, IVH \\
\hline
\end{tabular}

correlation, $\mathrm{p}<0.01$ ) as well as between cerebral perfusion pressure and cerebral blood flow velocity (Pearson correlation, $\mathrm{p}<0.05, r=0.54$; Spearman correlation, $\mathrm{p}<0.05, r=0.50$; fig 3 ). Their cerebrovascular autoregulatory capacity (cerebral autoregulation) as expressed by the $\mathrm{Mx}$ was also significantly reduced (independent $t$ test, $t=3.522 ; \mathrm{df}=38 ; \mathrm{p}<0.001$; table 2). There was no correlation between arterial blood pressure and cerebral blood flow velocity, or between cerebral perfusion pressure and cerebral blood flow velocity (fig 4), in patients with intact cerebrovascular reactivity (Pearson and Spearman correlation, NS). These results confirm our hypoth-

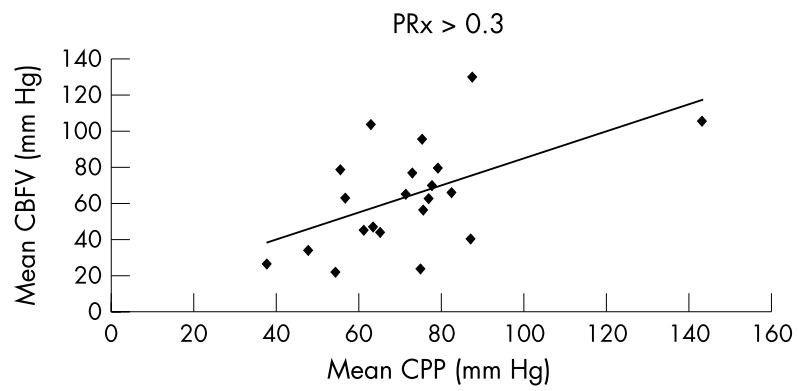

Figure 3 The significant correlation between mean cerebral perfusion pressure (CPP) and mean cerebral blood flow velocity (CBFV) in patients with impaired cerebrovascular reactivity (PRx $>0.3)$. esis. There was a significant overall correlation between PRx and $\mathrm{Mx}$ (Pearson correlation, $\mathrm{p}<0.007, r=0.42$; Spearman correlation, $\mathrm{p}<0.05 ; r=0.43$; fig 5).

The intracranial pressure, arterial blood pressure, cerebral perfusion pressure, and hemispheric cerebral autoregulation asymmetry did not differ in either group (table 2).

\section{DISCUSSION}

This study shows that the PRx is a valid index to monitor and quantify cerebrovascular reactivity in patients with head injuries. The positive correlation between pressure (arterial blood pressure and cerebral perfusion pressure) and flow (cerebral blood flow velocity) seen in patients with impaired or lost cerebrovascular reactivity shows that this intracranial pressure based index reflects changes in cerebral blood flow and cerebral autoregulatory capacity, which suggests a close link between intracranial flow and pressure in head injured patients. Although cerebral blood flow velocity measured with transcranial Doppler ultrasound in $\mathrm{cm} / \mathrm{s}$ does not measure cerebral blood volume flow in absolute terms, it is the only non-invasive monitoring tool available for a continuous approximation of cerebral blood flow in the intensive care setting. Transcranial Doppler ultrasound has been validated for this purpose and has become a widely accepted technique, used in numerous studies on cerebral autoregulation and

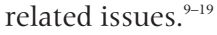


Table 2 Associated variables in patients with impaired cerebrovascular reactivity $(\mathrm{PRx}>0.3)$ and in those with intact cerebrovascular reactivity (PRx $<0.3)$

\begin{tabular}{|c|c|c|c|c|}
\hline \multirow[b]{2}{*}{ Variable } & \multicolumn{2}{|c|}{ Impaired CR $(n=21)$} & \multicolumn{2}{|c|}{ Intact CR $(n=19)$} \\
\hline & Mean (SD) & Range & Mean (SD) & Range \\
\hline$A B P$ & $87(22)$ & 60 to 160 & $89(11)$ & 78 to 113 \\
\hline CPP & 72 (21) & 38 to 143 & 75 (12) & 49 to 111 \\
\hline $\mathrm{ICP}$ & $16(7)$ & 5 to 25 & 14 (7) & 2 to 32 \\
\hline CBFV & $64(29)$ & 22 to 129 & 67 (19) & 43 to 104 \\
\hline PRx & $0.55(0.17)$ & 0.31 to 0.9 & $-0.01(0.24)$ & -0.59 to 0.25 \\
\hline$M x^{*}$ & $0.43(0.23)$ & -0.05 to 0.93 & $0.15(0.28)$ & -0.38 to 0.8 \\
\hline$\Delta \mathrm{l} / \mathrm{r} M \mathrm{x}$ & $0.16(0.25)$ & 0.07 to 1.01 & $0.16(0.29)$ & 0.01 to 1.28 \\
\hline
\end{tabular}

*Significant difference between impaired and intact $C R(p<0.001)$

$A B P$, mean arterial blood pressure $(\mathrm{mm} \mathrm{Hg}) ; C B F V$, cerebral blood flow velocity $(\mathrm{cm} / \mathrm{s}) ; C P P$, mean cerebral perfusion pressure $(\mathrm{mm} \mathrm{Hg})$; $C R$, cerebrovascular reactivity; ICP, intracranial pressure $(\mathrm{mm} \mathrm{Hg}) ; \mathrm{I} / \mathrm{r}$, left/right; $\mathrm{Mx}$, moving correlation coefficient between arterial blood pressure or cerebral perfusion pressure and cerebral blood flow velocity; Prx, index comparing arterial blood pressure and intracranial pressure.

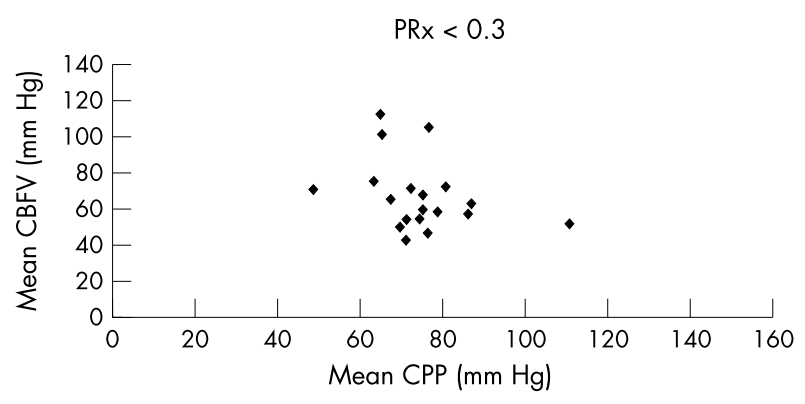

Figure 4 Absence of any correlation between mean cerebral perfusion pressure (CPP) and mean cerebral blood flow velocity $(\mathrm{CBFV})$ in patients with intact cerebrovascular reactivity $(\mathrm{PRx}<0.3)$.

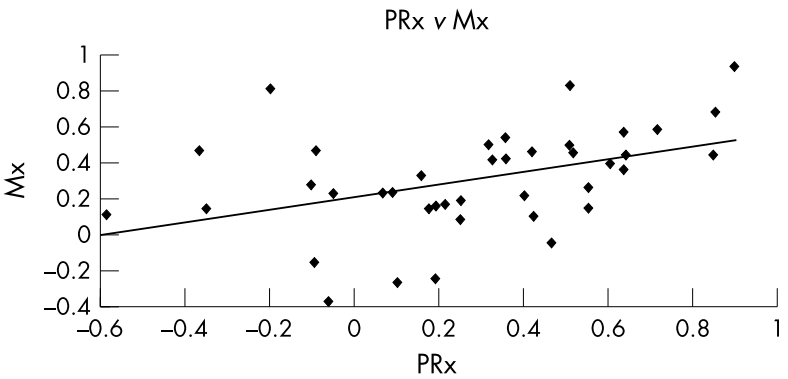

Figure 5 A scatterplot of $P R x \vee M x$ which confirms a significant correlation between these indices.

\section{Cerebrovascular reactivity and cerebral autoregulation} Activating intrinsic cerebral vasoconstrictor mechanisms by raising arterial blood pressure and hence the cerebral perfusion pressure in an attempt to reduce cerebral blood volume and thus intracranial pressure is appealing because it is apparently relatively easy to achieve in patients with intact cerebrovascular reactivity. The novelty of our study lies in its demonstration that cerebral blood flow velocity remains stable when arterial blood pressure/cerebral perfusion pressure varies in patients with preserved cerebrovascular reactivity, and that the blood flow velocity depends linearly on arterial blood pressure and cerebral perfusion pressure in those with impaired cerebrovascular reactivity. Our analysis confirms the previously reported correlation between PRx and Mx. ${ }^{20}$ Our data for cerebrovascular reactivity and cerebral autoregulation, calculated from identical datasets, also show that cerebral autoregulation is less well preserved in patients with impaired cerebrovascular reactivity. These finding supports the concept that cerebral autoregulation and cerebrovascular reactivity are closely linked but not identical. Steiner et al have pointed out ${ }^{7}$ that according to cerebral blood flow studies by
MacKenzie $^{21}$ "...the two expressions should not be used synonymously as vasodilatation reaches its maximum at arterial pressures below the lower threshold for constant cerebral blood flow.'

\section{Value of measuring cerebrovascular reactivity}

Steiner et al have shown that monitoring of cerebrovascular reactivity could identify an optimal cerebral perfusion pressure (CPP-OPT) in $60 \%$ of a large series of patients with traumatic brain injury. ${ }^{7}$ The use of cerebrovascular reactivitydirected rather than cerebral autoregulation-directed treatment in traumatic brain injury seems logical because cerebrovascular reactivity is derived from intracranial pressure, which is the main treatment target, rather than from cerebral blood flow velocity, which is used for cerebral autoregulation assessment. The authors stressed that at least 24 hours of continuous monitoring were required to determine CPP-OPT. ${ }^{7}$ This period is needed for accurate assessment of cerebrovascular reactivity over a sufficiently wide range of spontaneous fluctuations of mean arterial pressure. Based on our own study we cannot comment on the "optimal cerebral perfusion pressure" issue, as our monitoring time was too short to acquire data over sufficiently large variations in arterial blood pressure or cerebral perfusion pressure.

Oertel et al have recently reported that 55 induced hypertension tests in 26 head injured patients led to a reduction in intracranial pressure of more than $20 \%$ in only $6 \%$ of tests, whereas in $64 \%$ the intracranial pressure changed by no more than $20 \%$ from its baseline value, and in $31 \%$ there was an increase of more than $20 \%{ }^{22}$ They concluded that "...induced hypertension was consistently ineffective" for intracranial pressure control. We would suggest that continuous cerebrovascular reactivity monitoring in head injured patients such as the ones tested has great potential to identify the optimal cerebral perfusion pressure without additional hypertensive challenges. Also, their patients' mean (SD) arterial blood pressure before these tests was quite high at 104 (12) $\mathrm{mm} \mathrm{Hg}$, and the intracranial pressure was already well controlled and low at 16 (9) $\mathrm{mm} \mathrm{Hg}$. It is possible that the high cerebral perfusion pressure of $88 \mathrm{mmHg}$ was already close to, or in some cases even above, the optimal cerebral perfusion pressure. These factors may have confounded their interpretation of the value of induced hypertension for intracranial pressure control.

\section{Correlation with intracranial pressure, arterial blood pressure, cerebral perfusion pressure, and cerebral blood flow velocity}

In an earlier study, the Cambridge group reported that a positive PRx correlated with high intracranial pressure and episodes of arterial hypotension. ${ }^{4}$ We could not confirm this 
observation, but our monitoring time was shorter and only represents a snapshot image of a patient's entire course. Also, the intracranial pressure in both our groups was well controlled at the time of study, being above $25 \mathrm{~mm} \mathrm{Hg}$ in only two of the 40 patients. This further invalidates any formal comparison between their study and ours. The fact that intracranial pressure, arterial blood pressure, and cerebral perfusion pressure were all largely within the normal range probably did not allow us to establish a correlation between impaired cerebrovascular reactivity and arterial blood pressure or cerebral perfusion pressure, which are known to affect cerebrovascular reactivity and cerebral autoregulation. ${ }^{23}{ }^{24}$

Only three of 40 patients in our series had a mean cerebral blood flow velocity above $100 \mathrm{~cm} / \mathrm{s}$, a low threshold for cerebral vasospasm, and in only one was it over $120 \mathrm{~cm} / \mathrm{s} .{ }^{25}{ }^{26}$ It would have been interesting to assess whether cerebral blood flow velocity or vasospasm had an influence on cerebrovascular reactivity, which has not so far been reported. Yundt et al showed that parenchymal vessels distal to arteries with angiographic spasm after subarachnoid haemorrhage have abnormal static cerebral autoregulation. ${ }^{27}$ This clinical finding is supported by a study of subarachnoid haemorrhage in primates in which impairment of static cerebral autoregulation was found in chronic cerebral vasospasm..$^{28}$ Cerebral vasospasm is not common after traumatic brain injury, at least in the early stages, and any significant influence on cerebrovascular reactivity is unlikely unless vasospasm is severe enough to lead to a cerebral blood flow velocity of over $120 \mathrm{~cm} / \mathrm{s}$.

\section{Limitations and outlook}

Three issues are relevant to our data and its interpretation. First, the data on cerebrovascular reactivity were recorded only during the first five days after injury. There is evidence that cerebral autoregulation changes after injury and it is likely that this is also the case with cerebrovascular reactivity. The results of studies dealing with temporal profiles of cerebral autoregulation differ. While we have found that cerebral autoregulation gradually improves after traumatic brain injury, ${ }^{29}$ Czosnyka and coworkers showed significant improvement only during the first three days. ${ }^{5}$ Second, the effects of age on cerebrovascular reactivity after traumatic brain injury are unknown, and so far we have only found two references to the effects of age on cerebral autoregulation in healthy subjects. Vavilala et al, using the leg cuff deflation test, reported that cerebral autoregulation is less effective in normal adolescents aged 12 to 17 years than in adults aged 25 to $45 .^{30}$ Using the same test, Carey et al reported that cerebral autoregulation was not influenced by age, comparing two groups aged 29 (5) and 68 (5) years. ${ }^{31}$ Third, PRx is calculated using intracranial pressure which provides one dataset, whereas $\mathrm{Mx}$ is calculated using cerebral blood flow velocity recorded from both hemispheres which provides two datasets. While there was no formal right-left difference or asymmetry, our patients did not only have diffuse injuries; some had unilateral contusions and haematomas, where asymmetry has been shown to be significant. ${ }^{32}$ It would be interesting to study cerebrovascular reactivity using bilateral intracranial pressure recordings although it would be difficult to justify placing a second intracranial pressure monitor solely for research purposes in humans.

Monitoring of cerebrovascular reactivity using intracranial pressure measurements is possible only for as long as an intracranial pressure monitor is in use, which limits its application largely to a patient's time in the intensive care unit, although it would be interesting also to study cerebrovascular reactivity during recovery and follow up. As long as continuous non-invasive intracranial pressure monitoring remains unavailable, only monitoring of cerebral autoregulation-with continuous non-invasive arterial blood pressure and cerebral blood flow velocity recording-allows graded bedside assessment of cerebrovascular physiology in the later stages after traumatic brain injury. Based on the results of this and other studies it appears that assessment of cerebrovascular reactivity may also be useful for studies on cerebrovascular physiology outside the intensive care unit, in other patients whose intracranial pressure is being measured. This is most likely to be in patients with spontaneous subarachnoid haemorrhage or hydrocephalus.

\section{Conclusions}

The PRx, an index which quantifies the relation between arterial blood pressure or cerebral perfusion pressure and intracranial pressure, is valid for monitoring and quantifying cerebrovascular reactivity in patients with head injury, and offers the opportunity to study cerebrovascular physiology at the bedside. This index reflects changes in cerebral blood flow and cerebral autoregulatory capacity, which suggests a close link between blood flow and intracranial pressure in head injured patients. Our findings explain why elevation of arterial blood pressure or cerebral perfusion pressure may be useful for reducing intracranial pressure in selected head injured patients with intact cerebrovascular reactivity. Further clinical studies are needed to assess its value in other conditions with possibly disturbed cerebrovascular reactivity such as subarachnoid haemorrhage and hydrocephalus.

\section{ACKNOWLEDGEMENTS}

Our sincere thanks to the nursing staff of the intensive care unit at Westmead Hospital and the neurosurgical intensive care unit at Kiel for their support and cooperation in this investigation. EWL thanks Prof Randall N Chesnut of Portland, Oregon, USA for his support. This study was supported in part by the Westmead Hospital Charitable Trust No HREC2001/3/4.8(1185), Westmead, New South Wales, Australia, and in part by grant No La 916 2/1 from the Deutsche Forschungsgemeinschaft, Bonn, Germany.

\section{Authors' affiliations}

E W Lang, J Griffith, K Yip, A Yam, N W C Dorsch, Department of Neurosurgery, Westmead Hospital, University of Sydney, Westmead, New South Wales, Australia

J Lagopoulos, Department of Neurology, Westmead Hospital, University of Sydney

Y Mudaliar, Department of Intensive Care, Westmead Hospital,

University of Sydney

H M Mehdorn, Department of Neurosurgery,

Christian-Albrechts-Universität, Kiel, Germany

Competing interests: EW has received financial support for conference attendances from DWL, the manufacturer of the Doppler equipment, and B Braun, Australia, the distributor of the ICP device. EWL and JG run educational courses for Getz Bros, the distributor of the Doppler equipment in Australia, for which they charge a fee.

\section{REFERENCES}

1 Lang EW, Chesnut RM. A bedside method for investigating the integrity and critical thresholds of cerebral pressure autoregulation in severe traumatic brain injury patients. Br J Neurosurg 2000;14:117-26.

2 Lang EW, Chesnut RM. Intracranial pressure. Monitoring and management. Neurosurg Clin North Am 1994;5:573-605.

3 Rosner M. Pathophysiology and management of increased intracranial pressure. In: Andrews BT, ed. Neurosurgical intensive care. New York: McGraw Hill, 1993:57-112.

4 Czosnyka M, Smielewski $P$, Kirkpatrick $P$, et al. Continuous assessment of the cerebral vasomotor reactivity in head injury. Neurosurgery 1997;41:11-17.

5 Czosnyka M, Smielewski P, Kirkpatrick P, et al. Monitoring of cerebral autoregulation in head-injured patients. Stroke 1996;27:1829-34.

6 Bullock R, Chesnut RM, Clifton G, et al. Guidelines for the management of severe head injury. New York: Brain Trauma Foundation, 1996.

7 Steiner LA, Czosnyka M, Piechnik SK, et al. Continuous monitoring of cerebrovascular pressure reactivity allows determination of optimal cerebral perfusion pressure in patients with traumatic brain injury. Crit Care Med 2002; 30:733-8.

8 Piechnik SK, Yang X, Czosnyka M, et al. The continuous assessment of cerebrovascular reactivity: a validation of the method in healthy volunteers. Anesth Analg 1999:89:944-9.

9 Larsen FS, Olsen KS, Hansen BA, et al. Transcranial Doppler is valid for determination of the lower limit of cerebral blood flow autoregulation. Stroke 1994;25:1985-8. 
10 Lang EW, Mehdorn HM, Dorsch NW, et al. Continuous monitoring of cerebrovascular autoregulation: a validation study. J Neurol Neurosurg Psychiatry 2002;72:583-6.

11 Lang EW, Czosnyka M, Mehdorn HM. Tissue oxygen reactivity and cerebral autoregulation after severe traumatic brain injury. Crit Care Med 2003:31:267-71.

12 Lewis SB, Wong ML, Bannan PE, et al. Transcranial Doppler identification of changing autoregulatory thresholds after autoregulatory impairment. Neurosurgery 2001;48:369-75; discussion, 375-6.

13 Giller CA. A bedside test for cerebral autoregulation using transcranial Doppler ultrasound. Acta Neurochir (Wien) 1991;108:7-14.

14 Diehl RR, Linden D, Lucke D, et al. Phase relationship between cerebral blood flow velocity and blood pressure. A clinical test of autoregulation. Stroke 1995;26: 1801-4.

15 Dawson SL, Blake M, Panerai RB, et al. Dynamic but not static cerebral autoregulation is impaired in acute ischaemic stroke. Cerebrovasc Dis 2000; 10:126-32.

16 Daffertshofer M, Diehl RR, Ziems GU, et al. Orthostatic changes of cerebral blood flow velocity in patients with autonomic dysfunction. $J$ Neurol Sci 1991;104:32-8.

17 Czosnyka M, Smielewski P, Piechnik S, et al. Cerebral autoregulation following head injury. J Neurosurg 2001;95:756-63.

18 Chan KH, Miller JD, Dearden NM, et al. The effect of changes in cerebral perfusion pressure upon middle cerebral artery blood flow velocity and jugular bulb venous oxygen saturation after severe brain injury. J Neurosurg 1992;77:55-61.

19 Steinmeier R, Bauhuf C, Hubner U, et al. Continuous cerebral autoregulation monitoring by cross-correlation analysis: evaluation in healthy volunteers. Crit Care Med 2002;30:1969-75.

20 Czosnyka M, Smielewski P, Kirkpatrick P, et al. Continuous monitoring of cerebrovascular pressure-reactivity in head injury. Acta Neurochir Suppl (Wien) 1998;71:74-5

21 MacKenzie ET, Farrar JK, Fitch W, et al. Effects of hemorrhagic hypotension on the cerebral circulation. I. Cerebral blood flow and pial arteriolar caliber. Stroke 1979;10:711-18.
22 Oertel M, Kelly DF, Lee JH, et al. Efficacy of hyperventilation, blood pressure elevation, and metabolic suppression therapy in controlling intracranial pressure after head injury. J Neurosurg 2002;97:1045-53.

23 Wallis SJ, Firth J, Dunn WR. Pressure-induced myogenic responses in human isolated cerebral resistance arteries. Stroke 1996;27:2287-90.

24 Czosnyka M, Guazzo E, lyer V, et al. Testing of cerebral autoregulation in head injury by waveform analysis of blood flow velocity and cerebral perfusion pressure. Acta Neurochir Suppl (Wien) 1994;60:468-71.

25 Ringelstein RB. A practical guide to transcranial Doppler sonography. In: Noninvasive imaging of cerebrovascular disease. London: Alan R Liss, 1989:75-121.

26 Martin NA, Patwardhan RV, Alexander M, et al. Characterization of cerebral hemodynamic phases following severe head trauma: hypoperfusion, hyperemia, and vasospasm. J Neurosurg 1997;87:9-19.

27 Yundt KD, Grubb RL, Diringer MN, et al. Autoregulatory vasodilation of parenchymal vessels is impaired during cerebral vasospasm. J Cereb Blood Flow Metab 1998;18:419-24.

28 Takeuchi $\mathbf{H}$, Handa $Y$, Kobayashi $H$, et al. Impairment of cerebral autoregulation during the development of chronic cerebral vasospasm after subarachnoid hemorrhage in primates. Neurosurgery $1991 ; 28: 41-8$.

29 Lang EW, Yip K, Griffith J, et al. Hemispheric asymmetry and temporal profiles of cerebral pressure autoregulation in head injury. J Clin Neurosci (in press).

30 Vavilala MS, Newell DW, Junger E, et al. Dynamic cerebral autoregulation in healthy adolescents. Acta Anaesthesiol Scand 2002;46:393-7.

31 Carey BJ, Eames PJ, Blake M, et al. Dynamic cerebral autoregulation is unaffected by aging. Stroke 2000;31:2895-900.

32 Schmidt EA, Czosnyka M, Smielewski P, et al. Asymmetry of cerebral autoregulation following head injury. Acta Neurochir Suppl (Wien) 2002;81:133-4.

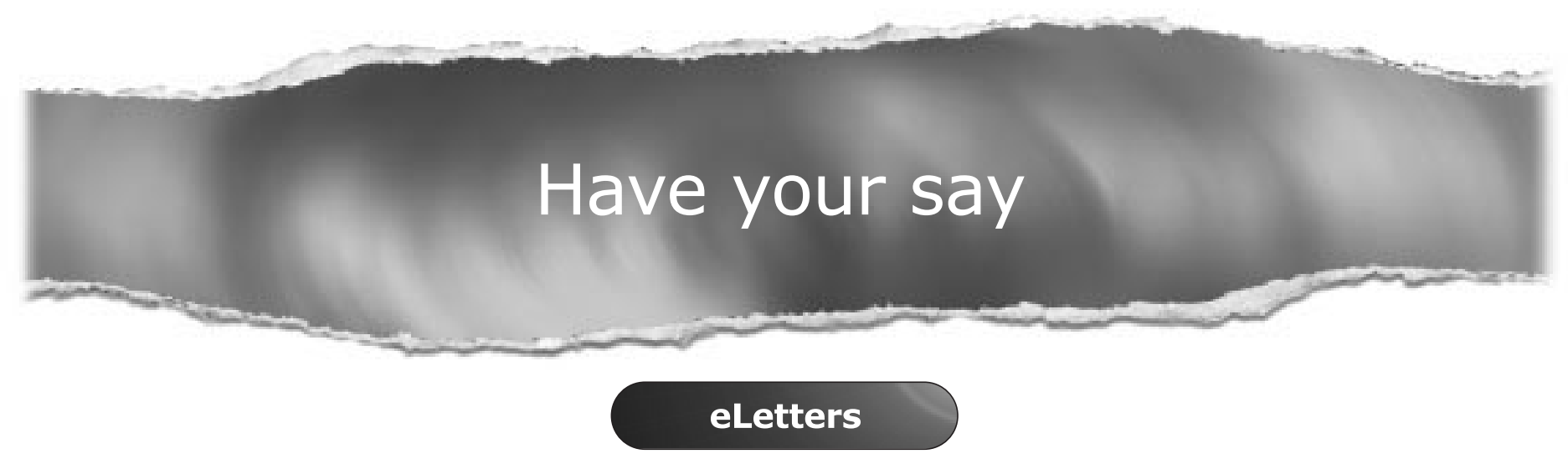

If you wish to comment on any article published in the Journal of Neurology, Neurosurgery, and Psychiatry

you can send an eLetter using the eLetters link at the beginning of each article. Your response will be posted on Journal of Neurology, Neurosurgery, and Psychiatry online within a few days of receipt (subject to editorial screening).

\section{www.jnnp.com}

